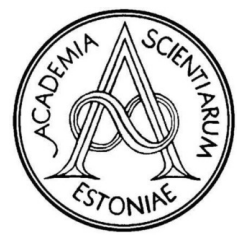

Proceedings of the Estonian Academy of Sciences,

$2021,70,2,155-162$

https://doi.org/10.3176/proc.2021.2.06

Available online at www.eap.ee/proceedings

\title{
Coequalizers and pullbacks in the category Seg of Segal topological algebras
}

\author{
Mart Abel \\ School of Digital Technologies, Tallinn University, Narva mnt. 25, 10120 Tallinn, Estonia \\ Institute of Mathematics and Statistics, University of Tartu, Narva mnt. 18,51009 Tartu, Estonia; mart.abel@tlu.ee, mart.abel@ut.ee \\ Received 24 November 2020, accepted 3 February 2021, available online 7 April 2021 \\ (c) 2021 Author. This is an Open Access article distributed under the terms and conditions of the Creative Commons Attribution- \\ NonCommercial 4.0 International License (http://creativecommons.org/licenses/by-nc/4.0/). \\ Abstract. In this paper we describe the coequalizers in the category Seg of Segal topological algebras and present some sufficient \\ conditions for the existence of pullbacks in Seg.
}

Key words: Segal topological algebras, category, coequalizer, pullback.

\section{INTRODUCTION}

Let $\mathbb{K}$ denote either the field $\mathbb{R}$ of real numbers or the field $\mathbb{C}$ of complex numbers. By a topological algebra we mean a topological vector space over $\mathbb{K}$ in which a separately continuous associative multiplication has been defined.

Let $\left(X, \tau_{X}\right)$ and $\left(Y, \tau_{Y}\right)$ be topological algebras over the field $\mathbb{K}$. We recall that their direct product $X \times Y=\{(x, y): x \in X, y \in Y\}$ is an algebra, if one defines the algebraic operations coordinate-wise, i.e.

$$
\left(x_{1}, y_{1}\right)+\left(x_{2}, y_{2}\right)=\left(x_{1}+x_{2}, y_{1}+y_{2}\right), \lambda\left(x_{1}, y_{1}\right)=\left(\lambda x_{1}, \lambda y_{1}\right) \text { and }\left(x_{1}, y_{1}\right)\left(x_{2}, y_{2}\right)=\left(x_{1} x_{2}, y_{1} y_{2}\right)
$$

for all $\lambda \in \mathbb{K},\left(x_{1}, y_{1}\right),\left(x_{2}, y_{2}\right) \in X \times Y$. The topology $\tau_{X \times Y}$ on $X \times Y$ is the product topology, i.e. its base is the collection

$$
\mathscr{B}_{X \times Y}=\left\{U \times V: U \in \tau_{X}, V \in \tau_{Y}\right\} .
$$

This topology makes $\left(X \times Y, \tau_{X \times Y}\right)$ a topological algebra over $\mathbb{K}$. Moreover, if $Z$ is a subalgebra of $X \times Y$, then we consider on $Z$ the subspace topology $\tau_{Z}=\left\{W \cap Z: W \in \tau_{X \times Y}\right\}$, which makes $\left(Z, \tau_{Z}\right)$ a topological algebra. In what follows, we will define the algebraic operations on the direct product of the two topological algebras $\left(X, \tau_{X}\right)$ and $\left(Y, \tau_{Y}\right)$ coordinate-wise and mean by the "subspace topology of the product topology of $\left(X, \tau_{X}\right)$ and $\left(Y, \tau_{Y}\right)$ " of the subalgebra $Z$ of $X \times Y$ the construction which gives $\tau_{Z}$ from $\tau_{X}$ and $\tau_{Y}$.

Now, let us recall the definition of a (general) Segal topological algebra, first published in [1].

A topological algebra $\left(A, \tau_{A}\right)$ is a left (right or two-sided) Segal topological algebra in a topological algebra $\left(B, \tau_{B}\right)$ via an algebra homomorphism $f: A \rightarrow B$, if 
(1) $\mathrm{cl}_{B}(f(A))=B$

(2) $\tau_{A} \supseteq\left\{f^{-1}(U): U \in \tau_{B}\right\}$, i.e. $f$ is continuous;

(3) $f(A)$ is a left (respectively, right or two-sided) ideal of $B$.

In short, we will denote a Segal topological algebra by a triple $(A, f, B)$.

For any category $\mathscr{C}$, we denote by $\mathrm{Ob}(\mathscr{C})$ the set of all objects of $\mathscr{C}$. For any $K, L \in \mathrm{Ob}(\mathscr{C})$, we denote by $\operatorname{Mor}(K, L)$ the set of all morphisms from $K$ to $L$.

As everything works similarly for left, right or two-sided Segal topological algebras, we will not mention the sideness in the paper. For better understanding, the reader can think about the left Segal topological algebras, right Segal topological algebras or two-sided Segal topological algebras, depending on which class of ideals the reader is more familiar with.

Let us continue by recalling the definition of the category Seg of all Segal topological algebras. The definition of Seg was first published in [4] together with the definition of another category of Segal topological algebras, called $\mathscr{S}(B)$, which has already been studied more thoroughly in several papers (see [2-9]). The category $\mathscr{S}(B)$ had all Segal topological algebras in the form $(A, f, B)$ as objects, where topological algebra $B$ was fixed and for any $(A, f, B),(C, g, B) \in \operatorname{Ob}(\mathscr{S}(B))$, the morphisms between $(A, f, B)$ and $(C, g, B)$ were all continuous algebra homomorphisms $\alpha: A \rightarrow C$ with the property $g \circ \alpha=f=1_{B} \circ f$, where $1_{B}: B \rightarrow B$ is an identity map on $B$. The category Seg has all Segal topological algebras as its objects. For any $(A, f, B),(C, g, D)$, the set $\operatorname{Mor}((A, f, B),(C, g, D))$ of morphisms from $(A, f, B)$ to $(C, g, D)$ consists of all such pairs $(\alpha, \beta)$ of continuous algebra homomorphisms $\alpha: A \rightarrow C$ and $\beta: B \rightarrow D$, for which $g \circ \alpha=\beta \circ f$. Hence, in case $(A, f, B),(C, g, D) \in \operatorname{Ob}(\mathbf{S e g})$ and $(\alpha, \beta) \in \operatorname{Mor}((A, f, B),(C, g, D))$, we have a commutative diagram

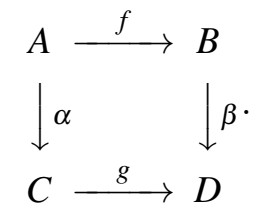

The composition of morphisms of $\mathbf{S e g}$ is defined componentwise as follows:

For any $(A, f, B),(C, g, D),(E, h, F) \in \mathrm{Ob}(\mathbf{S e g})$ and arbitrary morphisms $(\alpha, \beta):(A, f, B) \rightarrow(C, g, D)$, $(\gamma, \delta):(C, g, D) \rightarrow(E, h, F)$, the composition of $(\gamma, \delta)$ and $(\alpha, \beta)$ is $(\gamma, \delta) \circ(\alpha, \beta)=(\gamma \circ \alpha, \delta \circ \beta)$.

In [4], pp. 2-4, it was shown that this composition of morphisms is well-defined and associative. Moreover, it was demonstrated that the pair $\left(1_{A}, 1_{B}\right)$ of identity maps is the identity morphism for an object $(A, f, B)$ of Seg.

In [10], the study was started on the categorical properties of the category Seg. The present paper is the second article devoted to the more thorough study of the category Seg.

\section{COEQUALIZERS IN THE CATEGORY SEG}

We start this section with the definition of the coequalizer ${ }^{1}$ in the category Seg. For that, we need to generalize the definition of a coequalizer given in [4], pp. 8-9, in the case of the category $\mathscr{S}(B)$.

Definition 1. Let $(A, f, B)$ and $(C, g, D)$ be objects of the category Seg. The coequalizer of morphisms $\left(\alpha_{1}, \beta_{1}\right),\left(\alpha_{2}, \beta_{2}\right) \in \operatorname{Mor}((A, f, B),(C, g, D))$ is a pair $((Q, k, R) ;(\lambda, \mu))$ such that

(1) $(Q, k, R) \in \operatorname{Ob}(\mathbf{S e g})$ and $(\lambda, \mu) \in \operatorname{Mor}((C, g, D),(Q, k, R))$ with $\lambda \circ \alpha_{1}=\lambda \circ \alpha_{2}$ and $\mu \circ \beta_{1}=\mu \circ \beta_{2}$;

(2) for any pair $((S, l, T) ;(v, \rho))$ with $(S, l, T) \in \operatorname{Ob}(\mathbf{S e g})$ and $(v, \rho) \in \operatorname{Mor}((C, g, D),(S, l, T))$ with $v \circ \alpha_{1}=v \circ \alpha_{2}$ and $\rho \circ \beta_{1}=\rho \circ \beta_{2}$, there exists unique $(\sigma, \tau) \in \operatorname{Mor}((Q, k, R),(S, l, T))$ with $v=\sigma \circ \lambda$ and $\rho=\tau \circ \mu$ :

1 For the general definition of a coequalizer in an arbitrary category, see, e.g. [11], p. 64. 


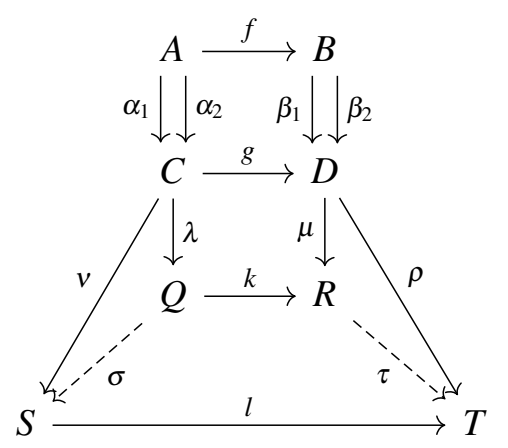

In what follows, we need to use the smallest two-sided ideal $I$ of $C$ generated by the set $M=\left\{\alpha_{1}(a)-\alpha_{2}(a): a \in A\right\}$. It is known that $I$ is equal to the set

$$
\left\{\sum_{k=1}^{n}\left(c_{k} m_{k} d_{k}+f_{k} m_{k}+m_{k} g_{k}+\lambda_{k} m_{k}\right): n \in \mathbb{Z}^{+}, c_{k}, d_{k}, f_{k}, g_{k} \in C, m_{k} \in M, \lambda_{k} \in \mathbb{K}\right\} .
$$

Similarly, we need to use the smallest two-sided ideal $J$ of $D$, generated by the set $N=\left\{\beta_{1}(b)-\beta_{2}(b): b \in B\right\}$, which is equal to the set

$$
\left\{\sum_{k=1}^{n}\left(c_{k} n_{k} d_{k}+f_{k} n_{k}+n_{k} g_{k}+\lambda_{k} n_{k}\right): n \in \mathbb{Z}^{+}, c_{k}, d_{k}, f_{k}, g_{k} \in D, n_{k} \in N, \lambda_{k} \in \mathbb{K}\right\} .
$$

On the sets $I$ and $J$ we will consider the subspace topologies $\tau_{I}$ and $\tau_{J}$, generated by the topologies $\tau_{A}$ of $A$ and $\tau_{B}$ of $B$, respectively, i.e. $\tau_{I}=\left\{U \cap I: U \in \tau_{A}\right\}$ and $\tau_{J}=\left\{V \cap J: V \in \tau_{B}\right\}$. In the theory of topological algebras, it is known that a quotient space of a topological algebra by its two-sided ideal is also a topological algebra when equipped with the quotient topology. Hence, $C / I$ and $D / J$, equipped with the quotient topologies, are topological algebras.

Theorem 1. Let $(A, f, B),(C, g, B) \in \operatorname{Ob}(\mathbf{S e g})$ and $\left(\alpha_{1}, \beta_{1}\right),\left(\alpha_{2}, \beta_{2}\right) \in \operatorname{Mor}((A, f, B),(C, g, D))$. Denote by $I$ the smallest two-sided ideal of $C$, generated by the set $M=\left\{\alpha_{1}(a)-\alpha_{2}(a): a \in A\right\}$, and by $J$ the smallest two-sided ideal of $D$, generated by the set $N=\left\{\beta_{1}(b)-\beta_{2}(b): b \in B\right\}$. Then the coequalizer of the morphisms $\left(\alpha_{1}, \beta_{1}\right),\left(\alpha_{2}, \beta_{2}\right)$ always exists and is the pair $((C / I, \tilde{g}, D / J) ;(p, q))$, where $p: C \rightarrow C / I$, $q: D \rightarrow D / J$ are the canonical projections, $C / I, D / J$ are equipped with the quotient topologies $\tau_{C / I}=\left\{V \subseteq C / I: p^{-1}(V) \in \tau_{C}\right\}, \tau_{D / J}=\left\{W \subseteq D / J: q^{-1}(W) \in \tau_{D}\right\}$, respectively, and $\tilde{g}: C / I \rightarrow D / J$ is defined by $\tilde{g}([c])=[g(c)]=q(g(c))$ for each $[c] \in C / I$.

Proof. Since $\left(\alpha_{1}, \beta_{1}\right),\left(\alpha_{2}, \beta_{2}\right) \in \operatorname{Mor}((A, f, B),(C, g, B))$, then

$$
g\left(\alpha_{1}(a)-\alpha_{2}(a)\right)=g\left(\alpha_{1}(a)\right)-g\left(\alpha_{2}(a)\right)=\beta_{1}(f(a))-\beta_{2}(f(a))
$$

for every $a \in A$. Hence, $g(M) \subseteq N$.

Take any $i \in I$. Then there exist $n_{i} \in \mathbb{Z}^{+}, m_{1}, \ldots, m_{n_{i}} \in M, \lambda_{1}, \ldots, \lambda_{n_{i}} \in \mathbb{K}$ and

$$
c_{1}, \ldots, c_{n_{i}}, d_{1}, \ldots, d_{n_{i}}, f_{1}, \ldots, f_{n_{i}}, g_{1}, \ldots, g_{n_{i}} \in C
$$

such that

$$
i=\sum_{k=1}^{n_{i}}\left(c_{k} m_{k} d_{k}+f_{k} m_{k}+m_{k} g_{k}+\lambda_{k} m_{k}\right) .
$$

As $g\left(m_{k}\right) \in N$ for every $m_{k} \in M$, then $g(i) \in J$. Hence, $g(I) \subseteq J$.

First, we will show that $(C / I, \tilde{g}, D / J) \in \mathrm{Ob}(\mathbf{S e g})$. 
We know that $C / I, D / J$, equipped with the quotient topologies, are topological algebras.

Let $c_{1}, c_{2} \in C$ such that $\left[c_{1}\right]=\left[c_{2}\right]$. Then $c_{1}-c_{2} \in I$ and $g\left(c_{1}-c_{2}\right) \in J$. Thus, $\tilde{g}\left(\left[c_{1}\right]\right)-\tilde{g}\left(\left[c_{2}\right]\right)=\left[g\left(c_{1}\right)\right]-\left[g\left(c_{2}\right)\right]=\left[g\left(c_{1}-c_{2}\right)\right]=\left[\theta_{D}\right]$, which means that $\tilde{g}\left(\left[c_{1}\right]\right)=\tilde{g}\left(\left[c_{2}\right]\right)$ and the map $\tilde{g}$ is well-defined. Moreover, $\tilde{g}$ is an algebra homomorphism because $g$ is an algebra homomorphism. Notice that $\tilde{g} \circ p=q \circ g$ because $(\tilde{g} \circ p)(c)=(q \circ g)(c)$ for each $c \in C$.

As $g(C)$ is a dense ideal in $D$, then for every $d \in D$ and every neighbourhood $O$ of $d$ there exists $c \in C$ such that $g(c) \in O$. Take any $[d] \in D / J$ and any neighbourhood $U$ of $[d]$ in $D / J$. Then there exists $W \in \tau_{D / J}$ such that $[d] \in W \subseteq U$. By the definition of the quotient topology $\tau_{D / J}$ we see that $q^{-1}(W)$ is an open neighbourhood of $d$ because $d \in q^{-1}(W)$ and $q^{-1}(W) \in \tau_{D}$. Hence, there exists $c \in C$ such that $g(c) \in q^{-1}(W)$. Now, $p(c)=[c] \in C / I$ is such an element of $C / I$, for which

$$
\tilde{g}([c])=q(g(c)) \in q\left(q^{-1}(W)\right) \subseteq W \subseteq U .
$$

Thus, for every $[d] \in D / J$ and every neighbourhood $U$ of $[d]$ in $D / J$ there exists $[c] \in C / I$ such that $\tilde{g}([c]) \in U$. This means that $\tilde{g}(C / I)$ is a dense subset of $D / J$.

Take any $W \in \tau_{D / J}$. Then $q^{-1}(W) \in \tau_{D}$, which means that there exists $U_{W} \in \tau_{D}$ such that $q^{-1}(W)=U_{W}$. As $(C, g, D) \in \mathrm{Ob}(\mathbf{S e g})$, it follows by the condition (2) of the definition of a Segal topological algebra that $g^{-1}\left(U_{W}\right) \in \tau_{C}$.

Now,

$$
\begin{aligned}
\tilde{g}^{-1}(W) & =\{[c]: \tilde{g}([c]) \in W\}=\{p(c): q(g(c)) \in W\} \\
& =\left\{p(c): c \in g^{-1}\left(q^{-1}(W)\right)\right\}=p\left(\left\{c: c \in g^{-1}\left(U_{W}\right)\right\}\right)=p\left(g^{-1}\left(U_{W}\right)\right) \in \tau_{C / I}
\end{aligned}
$$

because the projection $p$ is open and $g^{-1}\left(U_{W}\right) \in \tau_{C}$. Therefore, $\tau_{C / I} \supseteq\left\{\tilde{g}^{-1}(W): W \in \tau_{D / J}\right\}$.

Take any $x, y \in C / I, \lambda \in \mathbb{K}$ and $z \in D / J$. Then there exist $c_{x}, c_{y} \in C$ and $d_{z} \in D$ such that $x=\left[c_{x}\right]=p\left(c_{x}\right), y=\left[c_{y}\right]=p\left(c_{y}\right)$ and $z=\left[d_{z}\right]=q\left(d_{z}\right)$. Then $g\left(c_{x}\right), g\left(c_{y}\right) \in g(C)$. As $g(C)$ is a left (right or two-sided) ideal of $D$, then $g\left(c_{x}\right)+g\left(c_{y}\right), \lambda g\left(c_{x}\right), d_{z} g\left(c_{x}\right) \in g(C)$ (similarly, $g\left(c_{x}\right) d_{z} \in g(C)$ ). Thus, $\tilde{g}(x)+\tilde{g}(y)=\left[g\left(c_{x}\right)\right]+\left[g\left(c_{y}\right)\right]=\left[g\left(c_{x}\right)+g\left(c_{y}\right)\right], \lambda \tilde{g}(x)=\left[\lambda g\left(c_{x}\right)\right], z \tilde{g}(x)=\left[d_{z}\right]\left[g\left(c_{x}\right)\right]=\left[d_{z} g\left(c_{x}\right)\right] \in \tilde{g}(C / I)$ (similarly, $\tilde{g}(x) z \in \tilde{g}(C / I)$ ). Hence, $\tilde{g}(C / I)$ is an ideal of $D / J$ and $(C / I, \tilde{g}, D / J) \in \mathrm{Ob}(\mathbf{S e g})$.

It is known that $p$ and $q$, as canonical projections, are continuous algebra homomorphisms. Moreover, as $\alpha_{1}(a)-\alpha_{2}(a) \in M \subset I$ and $\beta_{1}(b)-\beta_{2}(b) \in N \subset J$, then $p\left(\alpha_{1}(a)\right)=p\left(\alpha_{2}(a)\right)$ for every $a \in A$ and $q\left(\beta_{1}(b)\right)=q\left(\beta_{2}(b)\right)$ for every $b \in B$. Hence, $p \circ \alpha_{1}=p \circ \alpha_{2}, q \circ \beta_{1}=q \circ \beta_{2}$ and the first condition of the coequalizer is fulfilled.

Suppose that there is $((S, l, T) ;(v, \rho))$ with $(S, l, T) \in \operatorname{Ob}(\mathbf{S e g})$ and $(v, \rho) \in \operatorname{Mor}((C, g, D),(S, l, T))$ with $v \circ \alpha_{1}=v \circ \alpha_{2}$ and $\rho \circ \beta_{1}=\rho \circ \beta_{2}$ :

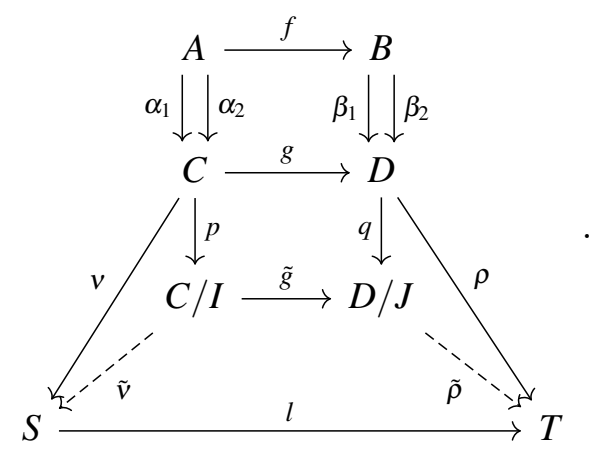

Consequently, we have $v\left(\alpha_{1}(a)-\alpha_{2}(a)\right)=\theta_{S}$ for every $a \in A$ and $\rho\left(\beta_{1}(b)-\beta_{2}(b)\right)=\theta_{T}$ for every $b \in B$. Hence, $v(I)=\left\{\theta_{S}\right\}$ and $\rho(J)=\left\{\theta_{T}\right\}$. Define the maps $\tilde{v}: C / I \rightarrow S$ by $\tilde{v}([c]):=v(c)$ for every $[c] \in C / I$ and $\tilde{\rho}: D / J \rightarrow T$ by $\tilde{\rho}([d]):=\rho(d)$ for every $[d] \in D / J$. The maps $\tilde{v}$ and $\tilde{\rho}$ are well-defined since $v(c)=v(d)$ for all $c_{1}, c_{2} \in C$, with $p\left(c_{1}\right)=p\left(c_{2}\right)$, and $\rho\left(d_{1}\right)=\rho\left(d_{2}\right)$ for all $d_{1}, d_{2} \in D$, with $q\left(d_{1}\right)=q\left(d_{2}\right)$. Notice that $\tilde{v}, \tilde{\rho}$ are continuous algebra homomorphisms because $v$ and $\rho$ are continuous algebra homomorphisms. 
Moreover, $(\tilde{v} \circ p)(c)=\tilde{v}([c])=v(c)$ for every $c \in C$ and $(\tilde{\rho} \circ q)(d)=\tilde{\rho}([d])=\rho(d)$ for every $d \in D$. Hence, $\tilde{v} \circ p=v$ and $\tilde{\rho} \circ q=\rho$. It is also clear that $\tilde{v}$ is the unique map with the property $\tilde{v} \circ p=v$ and $\tilde{\rho}$ is the unique map with the property $\tilde{\rho} \circ q=\rho$. Hence, the second condition of the coequalizer is also fulfilled and the pair $((C / I, \tilde{g}, D / J) ;(p, q))$ is the coequalizer of the morphisms $\left(\alpha_{1}, \beta_{1}\right)$ and $\left(\alpha_{2}, \beta_{2}\right)$.

\section{PULLBACKS IN THE CATEgORY SEG}

Similarly to the definition of the pullback ${ }^{2}$ in the category $\mathscr{S}(B)$ (see [4], pp. 10-11), we define also the pullback in the category Seg.

Definition 2. Let $(A, f, B),(C, g, D),(E, h, F) \in \operatorname{Ob}(\operatorname{Seg}), \quad(\alpha, \beta) \in \operatorname{Mor}((A, f, B),(E, h, F))$ and $(\gamma, \delta) \in \operatorname{Mor}((C, g, D),(E, h, F))$. An object $(P, j, Q)$ of the category Seg, together with morphisms $(\varepsilon, \zeta) \in \operatorname{Mor}((P, j, Q),(A, f, B))$ and $(\eta, \xi) \in \operatorname{Mor}((P, j, Q),(C, g, D))$, is called a pullback of morphisms $(\alpha, \beta)$ and $(\gamma, \delta)$, if

(1) $(\alpha, \beta) \circ(\varepsilon, \zeta)=(\gamma, \delta) \circ(\eta, \xi)$;

(2) for every $(R, k, S) \in \operatorname{Ob}(\mathbf{S e g})$ and $(\kappa, \lambda) \in \operatorname{Mor}((R, k, S),(A, f, B)),(\mu, v) \in \operatorname{Mor}((R, k, S),(C, g, D))$ such that $(\alpha, \beta) \circ(\kappa, \lambda)=(\gamma, \delta) \circ(\mu, v)$, there exists unique morphism $(\pi, \rho) \in \operatorname{Mor}((R, k, S),(P, j, Q))$ with $(\varepsilon, \zeta) \circ(\pi, \rho)=(\kappa, \lambda)$ and $(\eta, \xi) \circ(\pi, \rho)=(\mu, v)$

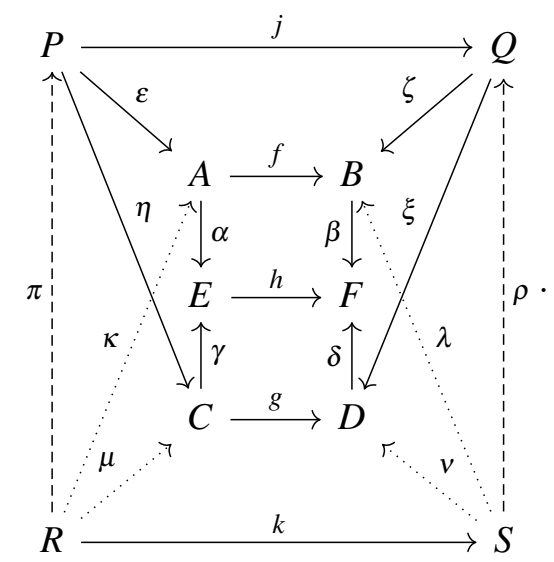

Using this definition of a pullback in Seg, we obtain the following result.

Proposition 1. Let $(A, f, B),(C, g, D),(E, h, F) \in \mathrm{Ob}(\mathbf{S e g}), \quad(\alpha, \beta) \in \operatorname{Mor}((A, f, B),(E, h, F))$ and $(\gamma, \delta) \in \operatorname{Mor}((C, g, D),(E, h, F))$. Suppose that the subset $F_{0}=(\beta \circ f)(A) \cap(\delta \circ g)(C)$ of $F$ is a dense ideal of $F$. Then the following claims hold:

(1) If $P=\left\{(a, c) \in A \times C: \alpha(a)=\gamma(c), h(\alpha(a)) \in F_{0}\right\}$,

$$
Q=\{(f(a), g(c)):(a, c) \in A \times C, \alpha(a)=\gamma(c)\} \cap \operatorname{cl}_{B \times D}\{(f(a), g(c)):(a, c) \in P\},
$$

$j((a, c))=(f(a), g(c))$, the topology on $P$ is the subspace topology of the product topology of $\left(A, \tau_{A}\right)$ and $\left(C, \tau_{C}\right)$, and the topology on $Q$ is the subspace topology of the product topology of $\left(B, \tau_{B}\right)$ and $\left(D, \tau_{D}\right)$, then the triple $(P, j, Q)$ is also an object of the category $\mathbf{S e g}$.

(2) The canonical projections $p_{A}: P \rightarrow A, p_{C}: P \rightarrow C, q_{B}: Q \rightarrow B$ and $q_{D}: Q \rightarrow D$ satisfy $\left(p_{A}, q_{B}\right) \in \operatorname{Mor}((P, j, Q),(A, f, B)),\left(p_{C}, q_{D}\right) \in \operatorname{Mor}((P, j, Q),(C, g, D))$ and

$$
(\alpha, \beta) \circ\left(p_{A}, q_{B}\right)=(\gamma, \delta) \circ\left(p_{C}, q_{D}\right) .
$$

\footnotetext{
2 For the general definition of a pullback in an arbitrary category, see, e.g. [11], p. 71.
} 
(3) If $\mathrm{cl}_{B \times D}\{(f(a), g(c)):(a, c) \in A \times C, \alpha(a)=\gamma(c)\}=\{(f(a), g(c)):(a, c) \in A \times C, \alpha(a)=\gamma(c)\}$, then $(P, j, Q)$, together with the maps $\left(p_{A}, q_{B}\right)$ and $\left(p_{C}, q_{D}\right)$, is the pullback of the morphisms $(\alpha, \beta)$ and $(\gamma, \delta)$.

Proof. (1) Let us first show that $P$ is a subalgebra of $A \times C$. For that, take any $\left(a_{1}, c_{1}\right),\left(a_{2}, c_{2}\right) \in P$ and $\lambda \in \mathbb{K}$. Then $\alpha\left(a_{1}\right)=\gamma\left(c_{1}\right), \alpha\left(a_{2}\right)=\gamma\left(c_{2}\right)$ and $h\left(\alpha\left(a_{1}\right)\right), h\left(\alpha\left(a_{2}\right)\right) \in F_{0}$. As $\alpha$ and $\beta$ are algebra homomorphisms, we have

$\alpha\left(a_{1}+a_{2}\right)=\alpha\left(a_{1}\right)+\alpha\left(a_{2}\right)=\gamma\left(c_{1}\right)+\gamma\left(c_{2}\right)=\gamma\left(c_{1}+c_{2}\right), \alpha\left(a_{1} a_{2}\right)=\alpha\left(a_{1}\right) \alpha\left(a_{2}\right)=\gamma\left(c_{1}\right) \gamma\left(c_{2}\right)=\gamma\left(c_{1} c_{2}\right)$

and

$$
\alpha\left(\lambda a_{1}\right)=\lambda \alpha\left(a_{1}\right)=\lambda \gamma\left(c_{1}\right)=\gamma\left(\lambda c_{1}\right) .
$$

As $h$ is an algebra homomorphism and $F_{0}$ is an ideal in $F$, it follows that

$$
h\left(\alpha\left(a_{1}+a_{2}\right)\right)=h\left(\alpha\left(a_{1}\right)\right)+h\left(\alpha\left(a_{2}\right)\right) \in F_{0}+F_{0} \subseteq F_{0}, h\left(\alpha\left(a_{1} a_{2}\right)\right)=h\left(\alpha\left(a_{1}\right)\right) h\left(\alpha\left(a_{2}\right)\right) \in F_{0} F_{0} \subset F_{0}
$$

and

$$
h\left(\alpha\left(\lambda a_{1}\right)\right)=h\left(\lambda \alpha\left(a_{1}\right)\right)=\lambda h\left(\alpha\left(a_{1}\right)\right) \in \lambda F_{0} \subseteq F_{0} .
$$

Hence, $\left(a_{1}, c_{1}\right)+\left(a_{2}, c_{2}\right) \in P,\left(a_{1}, c_{1}\right)\left(a_{2}, c_{2}\right) \in P$ and $\lambda\left(a_{1}, c_{1}\right) \in P$, which means that $P$ is a subalgebra of $A \times C$. As $P$ is equipped with the subspace topology $\tau_{P}$ of the product topology of $\left(A, \tau_{A}\right)$ and $\left(C, \tau_{C}\right)$, then $\left(P, \tau_{P}\right)$ is a topological algebra.

Similarly, one can see that the sets $\{(f(a), g(c)):(a, c) \in A \times C, \alpha(a)=\gamma(c)\},\{(f(a), g(c)):(a, c) \in P\}$ are subalgebras of $B \times D$. The closure of a subalgebra is still a subalgebra and the intersection of two subalgebras of the same algebra is a subalgebra. Hence, $Q$, equipped with the subspace topology, is a topological algebra.

As $Q \subseteq \operatorname{cl}_{B \times D}\{(f(a), g(c)):(a, c) \in P\}$ and $j(P)=\{(f(a), g(c)):(a, c) \in P\}$, it is clear that

$$
\operatorname{cl}_{Q}(j(P))=Q \cap \operatorname{cl}_{B \times D}(j(P))=Q,
$$

i.e. $j(P)$ is dense in $Q$.

As both $f$ and $g$ are continuous algebra homomorphisms, it is also clear that $j$ is a continuous algebra homomorphism.

In the same way we checked that $P$ is a subalgebra of $A \times C$, one can check that $j(P)$ is an ideal of the algebra $\{(f(a), g(c)):(a, c) \in A \times C, \alpha(a)=\gamma(c)\}$. Therefore, $j(P)$ is also an ideal of $Q$.

With that we have proved that $(P, j, Q)$ is an object of the category Seg.

(2) As projections, $p_{A}, p_{C}, q_{B}$ and $q_{D}$ are continuous algebra homomorphisms. Notice that

$$
\left(f \circ p_{A}\right)((a, c))=f(a)=q_{B}(f(a), g(c))=q_{B}(j(a, c))=\left(q_{B} \circ j\right)((a, c))
$$

and

$$
\left(g \circ p_{C}\right)((a, c))=g(c)=q_{D}\left((f(a), g(c))=q_{D}(j(a, c))=\left(q_{D} \circ j\right)((a, c)) \text { for every }(a, c) \in P .\right.
$$

Therefore, $f \circ p_{A}=q_{B} \circ j$ and $g \circ p_{C}=q_{D} \circ j$, which means that $\left(p_{A}, q_{B}\right) \in \operatorname{Mor}((P, j, Q),(A, f, B))$ and $\left(p_{C}, q_{D}\right) \in \operatorname{Mor}((P, j, Q),(C, g, D))$.

Notice that, for every $(a, c) \in\{(a, c) \in A \times C: \alpha(a)=\gamma(c)\}$, we have

$$
\left(\alpha \circ p_{A}\right)((a, c))=\alpha(a)=\gamma(c)=\left(\gamma \circ p_{C}\right)((a, c))
$$


and

$$
\begin{aligned}
& \left(\beta \circ q_{B}\right)((f(a), g(c)))=\left(\beta \circ q_{B}\right)(j((a, c)))=\beta\left(\left(q_{B} \circ j\right)((a, c))\right)=\beta\left(\left(f \circ p_{A}\right)((a, c))\right) \\
& \quad=(\beta \circ f)(a)=(h \circ \alpha)(a)=h\left(\left(\alpha \circ p_{A}\right)((a, c))\right)=h\left(\left(\gamma \circ p_{C}\right)((a, c))\right)=(h \circ \gamma)(c) \\
& \quad=(\delta \circ g)(c)=\delta(g(c))=\delta\left(q_{D}((f(a), g(c)))\right)=\left(\delta \circ q_{D}\right)((f(a), g(c))) .
\end{aligned}
$$

Hence, $\alpha \circ p_{A}=\gamma \circ p_{C}$ and $\beta \circ q_{B}=\delta \circ q_{D}$, which means that $(\alpha, \beta) \circ\left(p_{A}, q_{B}\right)=(\gamma, \delta) \circ\left(p_{C}, q_{D}\right)$.

(3) Based on the part (2) of the proof, we already know that the first condition of a pullback is satisfied.

Suppose that $(R, k, S) \in \operatorname{Ob}(\mathbf{S e g}),(\kappa, \lambda) \in \operatorname{Mor}((R, k, S),(A, f, B))$ and $(\mu, v) \in \operatorname{Mor}((R, k, S),(C, g, D))$ are such that $(\alpha, \beta) \circ(\kappa, \lambda)=(\gamma, \delta) \circ(\mu, v)$.

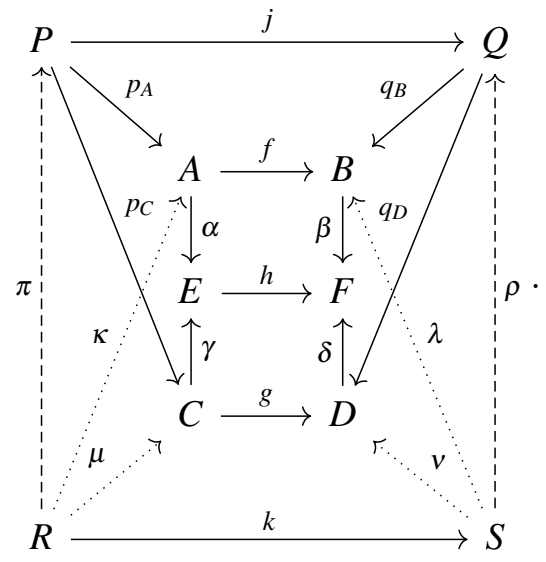

Notice that then $\alpha(\kappa(r))=\gamma(\mu(r))$ for every $r \in R$ and $\beta(\lambda(s))=\delta(v(s))$ for every $s \in S$. Moreover, from

$$
(\beta \circ f)(\kappa(r))=(h \circ \alpha)(\kappa(r))=h(\alpha(\kappa(r)))=h(\gamma(\mu(r)))=(h \circ \gamma)(\mu(r))=(\delta \circ g)(\mu(r)),
$$

it follows that $h(\alpha(\kappa(r))) \in(\beta \circ f)(A) \cap(\delta \circ g)(C)=F_{0}$, which means that $(\kappa(r), \mu(r)) \in P$ for every $r \in R$.

Take any $s \in S$. As $k(R)$ is dense in $S$, then there exists a net $\left(r_{i}\right)_{i \in I}$ of elements of $R$, such that the net $\left(k\left(r_{i}\right)\right)_{i \in I}$ converges to $s$. As $\lambda, v$ are continuous maps, then the net $\left(\lambda\left(k\left(r_{i}\right)\right)\right)_{i \in I}$ converges to $\lambda(s)$ and the net $\left(v\left(k\left(r_{i}\right)\right)\right)_{i \in I}$ converges to $v(s)$. Hence, the net $\left(\lambda\left(k\left(r_{i}\right)\right), v\left(k\left(r_{i}\right)\right)\right)_{i \in I}$ converges to $(\lambda(s), v(s))$. As $(\kappa, \lambda) \in \operatorname{Mor}((R, k, S),(A, f, B)),(\mu, v) \in \operatorname{Mor}((R, k, S),(C, g, D))$, then $\lambda \circ k=f \circ \kappa$ and $v \circ k=g \circ \mu$. Hence, the net $\left(f\left(\kappa\left(r_{i}\right)\right), g\left(\mu\left(r_{i}\right)\right)\right)_{i \in I}$ converges also to $(\lambda(s), v(s))$. By denoting $a_{i}=\kappa\left(r_{i}\right), c_{i}=\mu\left(r_{i}\right)$ for every $i \in I$, we see that $\left(a_{i}, c_{i}\right)=\left(\kappa\left(r_{i}\right), \mu\left(r_{i}\right)\right) \in P$ for every $i \in I$ (as shown above). Hence,

$$
\left(f\left(\kappa\left(r_{i}\right)\right), g\left(\mu\left(r_{i}\right)\right)\right) \in\{(f(a), g(c)):(a, c) \in P\} \subseteq\{(f(a), g(c)):(a, c) \in A \times C, \alpha(a)=\gamma(c)\}
$$

and

$$
(\lambda(s), v(s)) \in \operatorname{cl}_{B \times D}\{(f(a), g(c)):(a, c) \in A \times C, \alpha(a)=\gamma(c)\} \cap \operatorname{cl}_{B \times D}\{(f(a), g(c)):(a, c) \in P\} .
$$

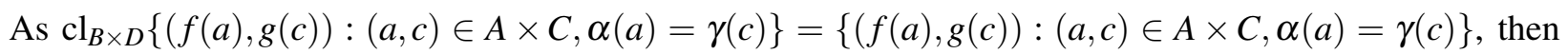
$(\lambda(s), v(s)) \in Q$ for every $s \in S$.

Define the maps $\pi: R \rightarrow P$ and $\rho: S \rightarrow Q$ by $\pi(r)=(\kappa(r), \mu(r))$ for every $r \in R$ and $\rho(s)=(\lambda(s), v(s))$ for every $s \in S$. Then $\pi$ and $\rho$ are continuous algebra homomorphisms, since $\kappa, \mu, \lambda$ and $v$ were continuous algebra homomorphisms.

Notice that

$$
(j \circ \pi)(r)=j(\kappa(r), \mu(r))=(f(\kappa(r)), g(\mu(r)))=(\lambda(k(r)), v(k(r)))=\rho(\kappa(r))=(\rho \circ k)(r)
$$

for every $r \in R$. Hence, $j \circ \pi=\rho \circ k$ and $(\pi, \rho) \in \operatorname{Mor}((R, k, S),(P, j, Q))$.

From the definitions of $p_{A}, p_{C}, q_{B}, q_{D}$ it is clear that $(\pi, \rho)$ is the unique morphism with $\left(p_{A}, q_{B}\right) \circ(\pi, \rho)=(\kappa, \lambda)$ and $\left(p_{C}, q_{D}\right) \circ(\pi, \rho)=(\mu, v)$. Hence, $(P, j, Q)$ is a pullback of the morphisms $(\alpha, \beta)$ and $(\gamma, \delta)$. 
Open questions. (1) Let $(A, f, B),(C, g, D),(E, h, F) \in \mathrm{Ob}(\mathbf{S e g}),(\alpha, \beta) \in \operatorname{Mor}((A, f, B),(E, h, F))$ and $(\gamma, \delta) \in \operatorname{Mor}((C, g, D),(E, h, F))$. Is any of the following two conditions necessary for the existence of a pullback of morphisms $(\alpha, \beta)$ and $(\gamma, \delta)$ ?

(a) $F_{0}=(\beta \circ f)(A) \cap(\delta \circ g)(C)$ is a dense ideal of $F$;

(b) $\{(f(a), g(c)):(a, c) \in A \times C, \alpha(a)=\gamma(c)\}$ is a dense subset of $B \times D$.

(2) In case at least one of the conditions of the open question (1) is not necessary, find the necessary and sufficient conditions for the existence of a pullback in the category Seg.

\section{CONCLUSION}

In the present paper we have shown that the coequalizers in the category Seg of Segal Topological Algebras always exist and found some sufficient conditions for the existence of pullbacks in the category Seg.

\section{ACKNOWLEDGEMENTS}

The research was supported by the institutional research funding PRG877 of the Estonian Ministry of Education and Research. The publication costs of this article were covered by the Estonian Academy of Sciences.

\section{REFERENCES}

1. Abel, M. Generalisation of Segal algebras for arbitrary topological algebras. Period. Math. Hung., 2018, 77(1), 58-68.

2. Abel, M. Initial, terminal and zero objects in the category $\mathscr{S}(B)$ of Segal topological algebras. Proceedings of the ICTAA 2018; Math. Stud. (Tartu), 2018, 7, 7-24.

3. Abel, M. About products in the category $\mathscr{S}(B)$ of Segal topological algebras. Proceedings of the ICTAA 2018; Math. Stud. (Tartu), 2018, 7, 25-32.

4. Abel, M. About some categories of Segal topological algebras. Poincare J. Anal. Appl., 2019, 1, 1-14.

5. Abel, M. Products and coproducts in the category $\mathscr{S}(B)$ of Segal topological algebras. Proc. Estonian Acad. Sci., 2019, 68(1), $88-99$.

6. Abel, M. About pushouts in the category $\mathscr{S}(B)$ of Segal topological algebras. Proc. Estonian Acad. Sci., 2019, 68(3), 319-323.

7. Abel, M. About the limits of inverse systems in the category $\mathscr{S}(B)$ of Segal topological algebras. Proc. Estonian Acad. Sci., 2020, 69(1), 1-10.

8. Abel, M. About the cocompleteness of the category $\mathscr{S}(B)$ of Segal topological algebras. Proc. Estonian Acad. Sci., 2020, 69(1), 53-56.

9. Abel, M. Coproducts in the category $\mathscr{S}(B)$ of Segal topological algebras, revisited. Period. Math. Hung., 2020, 81(2), $201-216$.

10. Abel, M. Initial objects, terminal objects, zero objects and equalizers in the category Seg of Segal topological algebras. Proc. Estonian Acad. Sci., 2020, 69(4), 361-367.

11. Mac Lane, S. Categories for the Working Mathematician. Graduate Texts in Mathematics, Vol. 5. Springer, New York, NY, 1971.

\section{Kovõrdsustajad ja tagasitõmbajad Segali topoloogiliste algebrate kategoorias Seg}

\section{Mart Abel}

On näidatud, et Segali topoloogiliste algebrate kategoorias Seg leiduvad alati kovõrdsustajad. Samuti on leitud piisavad tingimused tagasitõmbajate leidumiseks kategoorias Seg. 